\title{
Intermittency in solutions of the three-dimensional Navier-Stokes equations
}

\author{
By J. D. GIBBON ${ }^{1}$ AND CHARLES R. DOERING ${ }^{2}$ \\ ${ }^{1}$ Department of Mathematics, Imperial College London, London SW7 2BZ, UK \\ ${ }^{2}$ Department of Mathematics and Michigan Center for Theoretical Physics, University of Michigan, \\ 525 East University Avenue, Ann Arbor, MI 48109-1109, USA
}

(Received 28 October 2002 and in revised form 5 December 2002)

Dissipation-range intermittency was first observed by Batchelor \& Townsend (1949) in high Reynolds number turbulent flows. It typically manifests itself in spatio-temporal binary behaviour which is characterized by long, quiescent periods in the signal which are interrupted by short, active 'events' during which there are large excursions away from the average. It is shown that Leray's weak solutions of the three-dimensional incompressible Navier-Stokes equations can have this binary character in time. An estimate is given for the widths of the short, active time intervals, which decreases with the Reynolds number. In these 'bad' intervals singularities are still possible. However, the average width of a 'good' interval, where no singularities are possible, increases with the Reynolds number relative to the average width of a bad interval.

\section{Introduction}

More than 50 years have elapsed since Batchelor \& Townsend (1949) discovered the phenomenon of dissipation-range intermittency in various high Reynolds number turbulent flows. The main signature of an intermittent flow is that of long, relatively quiescent periods in the signal interspersed with short, dramatic events during which there are large excursions away from the average. Batchelor \& Townsend (1949) noted that large-wavenumber components were concentrated in isolated flow regions, which they referred to as 'spottiness'. They suggested that the energy associated with the small-scale components is distributed unevenly in space and roughly confined to regions which concomitantly become smaller with eddy size (see Kuo \& Corrsin 1971). In experimental investigations of the energy dissipation rate in several laboratory flows, and in the atmospheric surface layer, Meneveau \& Sreenivasan (1991) interpreted the evident intermittent nature of their signals in terms of multifractals.

Sreenivasan (1985) and Frisch (1995) (who has devoted a sizeable portion of his book to a discussion of mainly inertial-range intermittency) have both made the important point that the various turbulence models that have been popularly used over the years to explain intermittent phenomena make no direct appeal to the Navier-Stokes equations. Herein lies the mathematical challenge: to show that Leray's weak solutions of the Navier-Stokes equations typically have a temporal 'binary' character - to borrow a phrase from Sreenivasan (1985) - in which short, high-amplitude events associated with the fine structure are seen to be distinct from quieter, longer regions. Theoretically, the phenomenon of intermittency is a landmark to which many important questions lead. One such question is whether singularities occur during one or more of the intermittent events. Scheffer (1976) showed that the 
potentially singular set in time has zero half-dimensional Hausdorff measure, followed by Caffarelli, Kohn \& Nirenberg (1982) who showed that the singular set in spacetime has zero one-dimensional Hausdorff measure; for recent shorter proofs of this last result see Lin (1998) and Choe \& Lewis (2000). This means that if singularities do occur then they must be relatively rare events in space-time. The question of the existence of singularities is not only central to the difficult, unsolved problem of regularity (Temam 1983; Constantin \& Foias 1988; Doering \& Gibbon 1995) but it has long been suggested that it may have some bearing on the fractal nature of both the energy dissipation field and higher velocity derivatives; see, for instance, Mandelbrot (1976, 1982), Sreenivasan \& Meneveau (1988); Meneveau \& Sreenivasan (1991) and references in Frisch (1995).

In this paper some results on dissipation-range intermittency along the lines described above will be derived directly from Navier-Stokes equations. Explicit quantitative estimates are obtained, as a function of the Reynolds number $R e$, of the widths, and relative widths, of time intervals over which high-amplitude events can occur. Specifically, bounds are derived on the amount of time that certain small scales in the flow - time-dependent wavenumbers $\kappa_{n}(t)$ - are much larger than their typical size. These wavenumbers, defined precisely in the next section, are ratios of volume integrals taken over a finite periodic domain and are based on Navier-Stokes solutions. Even when Taylor's hypothesis has been used, experimental quantifications of intermittency have always been spatial, whereas the $\kappa_{n}(t)$ are functions only of time. Nevertheless, they are sensitive to the effects of spatial intermittency through volume integration; the finite domain prevents fluctuations from being averaged away. Clearly the $\kappa_{n}$ are not the same quantities as those measured by experimentalists, such as the energy dissipation rate, but estimates for the former are rigorous and make no appeal to any approximations. Physically they can be considered as a measure of the $2 n$th moment of the energy spectrum (see Doering \& Gibbon 2002). While the long time average of $\kappa_{n}$ has an upper bound proportional to $R e^{\lambda_{n}}$ (where $\lambda_{n}>0$ is an exponent also defined precisely below), excursions to high amplitudes - small scales where singularities could possibly occur - can only happen in relatively small time intervals, called 'bad' intervals of width $(\Delta t)_{b}$. During the intervening 'good' intervals of width $(\Delta t)_{g}$, the flow is smooth. In particular it will be shown that (i) the widths of the bad intervals $(\Delta t)_{b}$ are uniformly small at high Reynolds number, and (ii) the ratio of the average width of the good intervals, $\overline{(\Delta t}_{g}$, to the average width of the bad, $\overline{(\Delta t)}_{b}$, diverges as $R e \rightarrow \infty$. These are rigorous mathematical manifestations of the qualitative phenomenon of intermittency.

\section{Preliminaries and previous results}

Consider the three-dimensional incompressible Navier-Stokes equations for the velocity field $\boldsymbol{u}(\boldsymbol{x}, t)$

$$
\boldsymbol{u}_{t}+\boldsymbol{u} \cdot \nabla \boldsymbol{u}=v \Delta \boldsymbol{u}-\nabla p+\boldsymbol{f}(\boldsymbol{x}), \quad \nabla \cdot \boldsymbol{u}=0,
$$

on a periodic domain $\Omega=[0, L]^{3}$ where $p$ is the pressure and $v$ the viscosity. For convenience, a narrow-band, time-independent, divergence-free forcing function $\boldsymbol{f}(\boldsymbol{x})$ is used, with a preferred length scale $\ell$ related to that of the domain $L$ by $\ell=L / 2 \pi$ so that we will estimate $\|\boldsymbol{f}\|_{2} \approx \ell^{n}\left\|\nabla^{n} \boldsymbol{f}\right\|_{2}$. The notation \|\|$_{2}$ is the usual one for the $L^{2}$-norm. This choice of narrow-band forcing function with its restricted length scale simplifies, but does not restrict, the analysis (see Doering \& Gibbon 2002). The dimensionless control parameter is $G r=\ell^{3 / 2} v^{-2}\|f\|_{2}$; analysts conventionally call 
this the Grashof number. The root-mean-square velocity $U$ in the Reynolds number $R e=U \ell / \nu$ is defined via the time average of the energy, $U^{2}=L^{-3}\left\langle\|\boldsymbol{u}\|_{2}^{2}\right\rangle$, an a priori bounded quantity even for weak solutions (Leray 1934). The long time average is defined by

$$
\langle\Phi\rangle=\operatorname{LIM}_{t \rightarrow \infty}\left(\frac{1}{t} \int_{0}^{t} \Phi(s) \mathrm{d} s\right),
$$

where LIM is a generalized long-time limit for (weak) statistical solutions of the Navier-Stokes equations (Foias et al. 2001). Doering \& Foias (2002) have recently shown that for high $G r$, Navier-Stokes solutions obey $G r^{1 / 2} \leqslant c R e$, where $c$ is the first of many absolute dimensionless constants that enter the analysis ( $c$ is independent of $R e, L, v$ etc). Higher derivatives of the velocity field, together with those of the forcing, are incorporated into the set $(n \geqslant 1)$

$$
F_{n}=\int_{\Omega}\left(\left|\nabla^{n} \boldsymbol{u}\right|^{2}+\tau^{2}\left|\nabla^{n} \boldsymbol{f}\right|^{2}\right) \mathrm{d} V
$$

where the characteristic time $\tau$ is defined as $\tau=L^{2} \nu^{-1} G r^{-(1 / 2+\delta)}$ and $\delta$ is a small correction defined below. Ratios of the $F_{n}$ define a set of time-dependent 'wavenumbers', each with a dimension of inverse length

$$
\kappa_{n, r}(t)=\left(\frac{F_{n}}{F_{r}}\right)^{\frac{1}{2(n-r)}} .
$$

When $r=0$, it has been shown recently by Doering \& Gibbon (2002) that, in the asymptotic limit $R e \rightarrow \infty$, when $n \geqslant 1$, the $\kappa_{n, 0}$ obey

$$
L\left\langle\kappa_{n}\right\rangle \leqslant c_{n} R e^{\lambda_{n}}, \quad \lambda_{n}=3-\frac{5}{2 n}+\frac{\delta}{n},
$$

where the zero subscript in $\kappa_{n, 0}$ has been dropped for simplicity. We are free to choose the parameter $\delta$ in the range $0<\delta<\frac{1}{2}$; the constants $c_{n}$ depend only on $n$ and possibly on $\delta$ but not on $R e$. The form of $\tau$ has been chosen such that the forcing does not dominate over the fluid flow components. The case $n=1$ in inequality (2.5) is the equivalent of Leray's original result for weak solutions. For higher values of $n$ this remains a strictly weak solution result because the bound on the time average does not prevent integrable singularities in the $\kappa_{n}$. In addition, the $\kappa_{n}(t)$ are ordered for all $t$ such that

$$
L^{-1} \leqslant \kappa_{1} \leqslant \cdots \leqslant \kappa_{n} \leqslant \kappa_{n+1} \cdots
$$

An inequality exists for the $F_{n}$ of the form $F_{n}^{p+1} \leqslant F_{n-p} F_{n+1}^{p}$ for $0<p<n$ from which one obtains

$$
\left(\frac{\kappa_{n}}{\kappa_{n-p}}\right)^{n-p} \leqslant\left(\frac{\kappa_{n+1}}{\kappa_{n}}\right)^{p(n+1)} .
$$

Ratios of the $\kappa_{n}$ arose in Batchelor \& Townsend (1949) who were concerned with experimental measurements of the ratio $D_{6} D_{4}^{-2} D_{2}$ : to within constants their $D_{2 n}$ are equivalent to the $F_{n}$ defined in equation (2.3) with $f=0$. Hence $D_{6} D_{4}^{-2} D_{2}$ is similar to

$$
F_{3} F_{2}^{-2} F_{1}=\left(\frac{\kappa_{3}}{\kappa_{2}}\right)^{6}\left(\frac{\kappa_{2}}{\kappa_{1}}\right)^{-2} .
$$

Their experimental results showed that $D_{6} D_{4}^{-2} D_{2}$ was larger than would normally be expected for Gaussian data. 


\section{The binary form of the time-axis and intermittency}

The measurements of Batchelor \& Townsend (1949) provide us with the motivation for examining the ratio $\kappa_{n+1} / \kappa_{n}$ to investigate the binary nature of the time axis. Take two parameters $0<\mu<1$ and $0<\alpha<1$ such that $\mu+\alpha=1$. The inverses $\mu^{-1}$ and $\alpha^{-1}$ will be used as exponents in the Hölder inequality on the far right-hand side of

$$
\left\langle\kappa_{n}^{\alpha}\right\rangle \leqslant\left\langle\kappa_{n+1}^{\alpha}\right\rangle=\left\langle\left(\frac{\kappa_{n+1}}{\kappa_{n}}\right)^{\alpha} \kappa_{n}^{\alpha}\right\rangle \leqslant\left\langle\left(\frac{\kappa_{n+1}}{\kappa_{n}}\right)^{\alpha / \mu}\right\rangle^{\mu}\left\langle\kappa_{n}\right\rangle^{\alpha}
$$

thereby giving

$$
\left\langle\left(\frac{\kappa_{n+1}}{\kappa_{n}}\right)^{\alpha / \mu}\right\rangle \geqslant\left(\frac{\left\langle\kappa_{n}^{\alpha}\right\rangle}{\left\langle\kappa_{n}\right\rangle^{\alpha}}\right)^{1 / \mu}=\left\langle\kappa_{n}^{\alpha}\right\rangle\left(\frac{\left\langle\kappa_{n}^{\alpha}\right\rangle}{\left\langle\kappa_{n}\right\rangle}\right)^{\alpha / \mu} .
$$

Navier-Stokes information can be injected into these formal manipulations by using the weak solution bound (2.5) and the lower bound $L \kappa_{n} \geqslant 1$ in the ratio on the far right-hand side to give

$$
\left\langle\left[c_{n}\left(\frac{\kappa_{n+1}}{\kappa_{n}}\right)\right]^{\alpha / \mu}-\left[\left(L \kappa_{n}\right)^{\mu} R e^{-\lambda_{n}}\right]^{\alpha / \mu}\right\rangle \geqslant 0
$$

with the same $c_{n}$ as in (2.5). A positive time average implies that the integrand is positive on some time intervals (at least one) but not necessarily the whole $t$-axis. The intervals where this integrand is positive are designated as good intervals on which the inequality

$$
c_{n}\left(\frac{\kappa_{n+1}}{\kappa_{n}}\right) \geqslant\left(L \kappa_{n}\right)^{\mu} R e^{-\lambda_{n}}
$$

holds. On the rest of the time-axis, designated as bad intervals, the $\kappa_{n}$ must satisfy the reverse inequality

$$
c_{n}\left(\frac{\kappa_{n+1}}{\kappa_{n}}\right) \leqslant\left(L \kappa_{n}\right)^{\mu} R e^{-\lambda_{n}} .
$$

Clearly this allows the $t$-axis to display a 'binary' character. This is not an absolute necessity, however, for there are steady, laminar flows for which the whole $t$-axis would be a good interval. The precise distribution and occurrence of the good/bad intervals on the $t$-axis remains an open question but the analysis in this paper will concentrate on flows that have a binary character.

On bad intervals, where the $\kappa_{n}$ satisfy inequality (3.5), use of (2.6) shows that $\kappa_{n}$ must be bounded below uniformly by

$$
L \kappa_{n}(t) \geqslant c_{n, \mu} R e^{\lambda_{n} / \mu}
$$

where $c_{n, \mu}=c_{n}^{1 / \mu}$. Depending on the choice of $\mu<1$, and for $R e \rightarrow \infty$, it is clear that this lower bound is considerably larger, possibly by many orders of magnitude, than the upper bound on the global time average (2.5). Note that (3.5) itself does not imply that a bad interval is finite, or that bad intervals are short in width, or even that their widths are short relative to the good intervals. To prove these results requires the use of (3.6) in combination with Navier-Stokes inequalities on $\kappa_{n, 1}$ as in the Appendix. An upper bound is found there on the width $(\Delta t)_{b}=t_{1}-t_{0}$ of a bad interval

$$
(\Delta t)_{b} \leqslant c_{n}^{(1)} L^{2} v^{-1} R e^{4-\lambda_{n} / \mu}
$$


with the requirement that $\mu$ be restricted to lie in a range narrower than $0<\mu<1$, namely

$$
0<\mu<\lambda_{n} / 4
$$

If $\mu$ lies outside this range then the methods employed fail to find any finite upper bound on $(\Delta t)_{b}$. The range of $\mu$ given in (3.8) shows that the exponent of $R e$ in (3.7) is clearly negative, which means that the bad intervals decrease with increasing Re. Importantly, as the Appendix shows, within these bad intervals no upper bound on the $\kappa_{n}$ has been found. Hence, despite their finite width, and reinforced by the size of their lower bound given in (3.6), singularity formation cannot be ruled out.

What happens on the good intervals for this range of $\mu$ ? It is not difficult to turn the standard differential inequality for the $F_{n}$ into one for the $\kappa_{n}$ (Doering \& Gibbon 2001):

$$
n \dot{\kappa}_{n} \leqslant-v\left[\left(\frac{\kappa_{n+1}}{\kappa_{n}}\right)^{2 n+2}-1\right] \kappa_{n}^{3}+c_{n} \kappa_{n}^{7 / 2} F_{0}^{1 / 2}+\operatorname{Re} \kappa_{n} .
$$

Inequality (3.9) illustrates the regularity problem: for large $\kappa_{n}$ the negative $\kappa_{n}^{3}$ viscous term is not strong enough to control the $\kappa_{n}^{7 / 2}$ term despite the fact that $F_{0}$ is always a bounded function. Alternatively, if (2.7) is used together with (2.6), it can be shown that if any $\kappa_{n}$ is bounded then all $\kappa_{n}$ are bounded; it ought to be emphasized, however, that no a priori upper bound on any $\kappa_{n}$ is known to exist for arbitrary long times.

On the good intervals, however, (3.4) comes to our aid when applied to (3.9). Its effect is to add power to the $\kappa_{n+1} / \kappa_{n}$ term at fine scales when $L \kappa_{n}>c_{n, \mu} R e^{\lambda_{n} / \mu}$. No singularities can occur on these intervals provided

$$
\mu>\frac{1}{4(n+1)} \text {. }
$$

In combination with (3.8), this shows that the range in which $\mu$ must lie is

$$
\frac{1}{4(n+1)}<\mu<\frac{\lambda_{n}}{4}
$$

which narrows with decreasing $n$. The distribution of the good/bad intervals on the $t$-axis may differ for each $n$. If a point $t=t^{*}$ lies in a bad interval corresponding to $\kappa_{n}$ for some $n$ but also lies in a good interval of another $\kappa_{m}(m \neq n)$, then all $\kappa_{n}$ for every $n$ are bounded at $t^{*}$. A singularity is only possible if $t^{*}$ lies in a bad interval for every $n \geqslant 1$.

It is clearly desirable to have a lower bound on the width of the good intervals $(\Delta t)_{g}$ that increases relative to $(\Delta t)_{b}$. We have not been able to show that the width of each good interval is large with respect to each of the bad intervals. However, an elementary argument based on the Markov-Chebychev inequality shows that the average width of a good interval increases relative to the average width of a bad interval. Consider an interval of time $\left[t_{p}, t_{q}\right]$ that contains the same number $N$ of good and bad intervals of widths $(\Delta t)_{g}^{(i)}$ and $(\Delta t)_{b}^{(i)}$ respectively. Then the fraction of time occupied by the bad intervals satisfies 


$$
\begin{aligned}
\frac{\sum_{i=1}^{N}(\Delta t)_{b}^{(i)}}{\sum_{i=1}^{N}\left[(\Delta t)_{g}^{(i)}+(\Delta t)_{b}^{(i)}\right]} & \leqslant \frac{1}{t_{q}-t_{p}} \int_{\left[L \kappa_{n}(t) \geqslant c_{n, \mu} R e^{\lambda_{n} / \mu}\right] \cap\left[t_{p}, t_{q}\right]} \mathrm{d} t \\
& \leqslant \frac{1}{t_{q}-t_{p}}\left(\frac{\int_{\left[t_{p}, t_{q}\right]} L \kappa_{n} \mathrm{~d} t}{c_{n, \mu} R e^{\lambda_{n} / \mu}}\right) .
\end{aligned}
$$

Now we define the average widths of the good and bad intervals as

$$
{\overline{(\Delta t)_{g}}}_{=} \lim _{N \rightarrow \infty} \frac{1}{N} \sum_{i=1}^{N}(\Delta t)_{g}^{(i)}, \quad \overline{(\Delta t}_{b}=\lim _{N \rightarrow \infty} \frac{1}{N} \sum_{i=1}^{N}(\Delta t)_{b}^{(i)},
$$

so as $N \rightarrow \infty$ and $t_{q}-t_{p} \rightarrow \infty$, we have

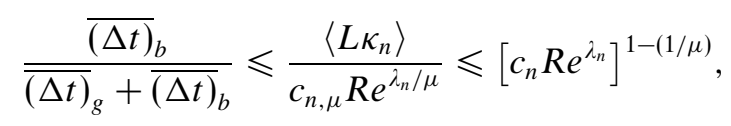

where we have used (3.6) and (2.5). Hence

$$
\overline{(\Delta t)}_{g} \geqslant \overline{(\Delta t)}_{b}\left\{\left[c_{n} R e^{\lambda_{n}}\right]^{(1 / \mu)-1}-1\right\},
$$

and so the ratio of $\overline{(\Delta t)}_{g}$ to $\overline{(\Delta t)}_{b}$ diverges as $R e \rightarrow \infty$ because $\mu<1$.

What is the nature of solutions on both these intervals and how close is the binary behaviour described in this section to the observed phenomenon of intermittency? In the bad intervals, where large lower bounds indicate high activity, singularities cannot be ruled out, but the precise nature of weak solutions there remains an open question. The factor of $\mu^{-1}$ in the exponent in (3.6) is significant: at the lower end of the range in which $\mu$ lies, the $\mu^{-1}$ factor magnifies the lower bound, effectively pushing up the value of $\kappa_{n}$ to heights many orders of magnitude above the global weak solution time average in (2.5). So as not to violate this average the $\kappa_{n}$ must behave in the good intervals is such a manner that they balance the much larger than average behaviour within the bad intervals. While this indicates a relative degree of quiescence it is hard to prove this in a more specific manner; the situation is also complicated by the fact that the 'good' solution must match onto the 'bad' (weak) solution at $t_{0}$ and $t_{1}$ where they must both be $O\left(R e^{\lambda_{n} / \mu}\right)$. A perusal of (3.9) shows that values of $\kappa_{n}$ can indeed be this large. What happens in the middle section of the good intervals is therefore an important question. Although singularities are ruled out in these intervals it would be gratifying to be able to prove that solutions there are flatter than average. These two open questions regarding potential singularities and the quiescence of solutions in the regions surrounding them are not independent.

\section{Conclusion}

This paper has attempted to address the issue of how time intermittency can arise in the Navier-Stokes variables $\kappa_{n}$ when the domain is finite and periodic. The obvious connection with the well-known regularity problem for the Navier-Stokes equations is of interest. It has been known for many decades that they are regular for arbitrarily long times for small initial data. For arbitrarily large initial data, regularity for short times is all that is known. The books by Temam (1983) and Constantin \& Foias (1988) give a fuller story, including work on weak solutions. The picture that the 
results of this paper presents is that of a 'punctured time-axis'. Solutions of the Navier-Stokes equations turn out to be regular on longer, good intervals which are themselves interspersed with shorter holes of finite but small width in the axis (the bad intervals) where regularity is uncertain. The widths of the holes decrease as $R e \rightarrow \infty$, whereas the widths of the good intervals increase relative to the latter in the averaged sense. The controlling mechanism within the good intervals is that of the viscous term effectively increasing sufficiently in strength at fine scales to control the nonlinear terms. Interestingly, the $\kappa_{n}$ are forced to have large lower bounds $L \kappa_{n} \geqslant c_{n, \mu} R e^{\lambda_{n} / \mu}$ within the bad intervals where activity is high.

It is here where it is tempting to investigate the suggested connection between the multifractal nature of intermittent flows and either singular or near-singular behaviour, as discussed by many authors such as Sreenivasan \& Meneveau (1988) and Meneveau \& Sreenivasan (1991). This raises the question of whether the binary nature of the $\kappa_{n}$ on the time-axis is an artefact of the analysis or whether it is real. Leray (1934) showed that the singular set in time $S$ has zero-dimensional Lebesgue measure, which means that if singularities occur then they must do so at points. Scheffer (1976) took this a stage further and showed that $S$ has zero half-dimensional Hausdorff measure. To prove true fractality of solutions by the methods of this paper it would be necessary to show that each bad interval itself has an internal binary structure leading to a repetitively embedded binary sub-structure at every scale. To see whether it is possible to prove this, the calculation performed in (3.2) can be repeated with the time integrals taken only over the finite interval $(\Delta t)_{b}$ :

$$
\int_{(\Delta t)_{b}}\left(\frac{\kappa_{n+1}}{\kappa_{n}}\right)^{\alpha / \mu} \mathrm{d} t \geqslant\left(\int_{(\Delta t)_{b}} \kappa_{n}^{\alpha} \mathrm{d} t\right)\left(\frac{\int_{(\Delta t)_{b}} \kappa_{n}^{\alpha} \mathrm{d} t}{\int_{(\Delta t)_{b}} \kappa_{n} \mathrm{~d} t}\right)^{\alpha / \mu} .
$$

To find a lower bound on the ratio in the round brackets on the far-right hand leads to the necessity of finding a lower bound on $(\Delta t)_{b}$ and it is this that is currently unavailable. If, however, such a lower bound could be found then it is possible that the process could then be repeated ad infinitum, thereby producing the sieve-like structure of a Cantor-like set within the bad intervals.

Finally the a priori bounds on the $\left\langle\kappa_{n}\right\rangle$ in (2.5) are higher than the conventional Kolmogorov theory of turbulence predicts and are consistent with a $k^{-8 / 3}$ spectrum (Doering \& Gibbon 2002; Sulem \& Frisch 1975). If the conventional picture may be caricatured as a wide $k^{-5 / 3}$ inertial range with a sharp cut-off at the Kolmogorov scale $\sim L R e^{-3 / 4}$, then the $\left\langle\kappa_{n}\right\rangle$ would be expected to scale with a different $\lambda_{n}$, denoted as $\tilde{\lambda}_{n}$, such that

$$
L\left\langle\kappa_{n}\right\rangle \sim c_{n} \operatorname{Re}^{\tilde{\lambda}_{n}}, \quad \tilde{\lambda}_{n}=\frac{3}{4}-\frac{1}{4 n}+\frac{\delta}{n} .
$$

Although it has not been proved that these exponents are rigorous bounds - at least not without intermittency-suppressing assumptions; see Doering \& Gibbon (2002) such scaling is not inconsistent with the intermittency picture described here. Indeed, this kind of scaling in the $\left\langle\kappa_{n}\right\rangle$ does not rule out singularities in the solutions during the bad time intervals but reduces the length scale below which these occur to roughly the Kolmogorov length. The dynamics associated with these intervals would be the cause of sub-Kolmogorov fine structure in the flow; the widths of these intervals would vanish uniformly as $R e^{4-\tilde{\lambda}_{n} / \mu}$ for some $0<\mu<\tilde{\lambda}_{n} / 4$, as in (3.7) and (3.8). Moreover, the ratio of the widths of the good to bad time intervals would still diverge as in (3.15). 
C.R.D. would like to acknowledge support from the US National Science Foundation.

\section{Appendix. An estimate for $(\Delta t)_{b}$}

Take the differential inequality for $\kappa_{n, 1}$ (Doering \& Gibbon 1995) and divide by $\kappa_{n, 1}^{2}$ :

$$
(n-1) \frac{\mathrm{d} \kappa_{n, 1}}{\mathrm{~d} t} \leqslant-v\left(\frac{\kappa_{n+1,1}}{\kappa_{n, 1}}\right)^{2 n} \kappa_{n, 1}^{3}+v \kappa_{n, 1} \kappa_{2,1}^{2}+c_{n} \kappa_{n, 1}^{5 / 2} F_{1}^{1 / 2}+v L^{-2} \operatorname{Re} \kappa_{n, 1} .
$$

Using Young's inequality to break up the $\kappa_{n, 1}^{1 / 2} F_{1}^{1 / 2}$ and $\kappa_{n+1,1} \geqslant \kappa_{n, 1}$, (A 1$)$ gives

$$
(n-1) \frac{\mathrm{d}}{\mathrm{d} t} \kappa_{n, 1}^{-1} \geqslant \frac{1}{2} \nu \kappa_{n, 1}-v \kappa_{2,1}-c_{n} \nu^{-1} F_{1}-v L^{-2} \operatorname{Re} \kappa_{n, 1}^{-1} .
$$

Using the lower bound $L \kappa_{n, 1} \geqslant L \kappa_{n} \geqslant R e^{\lambda_{n} / \mu}$ and integrating from $t_{0} \rightarrow t$ gives

$$
\frac{A_{n}}{\nu}\left[\kappa_{n, 1}^{-1}(t)-\kappa_{n, 1}^{-1}\left(t_{0}\right)\right] \geqslant \int_{t_{0}}^{t}\left\{\kappa_{n, 1}\left(t^{\prime}\right)-2 L^{-1} R e^{1-\lambda_{n} / \mu}-2 \kappa_{2,1}-2 c_{n} v^{-2} F_{1}\right\} \mathrm{d} t^{\prime},
$$

where $A_{n}=2(n-1)$. Now it is necessary to find estimates for the time integrals of $F_{1}$ and $\kappa_{2,1}$ over the interval $\Delta t=t-t_{0}$. The first comes from integrating the energy inequality for weak solutions from $t_{0} \rightarrow t$ and requires the use of the global pointwise upper bound for the energy $\|\boldsymbol{u}\|_{2}$. This gives

$$
\int_{t_{0}}^{t} F_{1}\left(t^{\prime}\right) \mathrm{d} t^{\prime} \leqslant c_{1} v L R e^{4}+L^{-1} v^{2}\left(c_{2} R e^{4}+c_{3} R e^{2}\right)(\Delta t) .
$$

The second comes from manipulating the inequality

$$
\frac{1}{2} \dot{F}_{1} \leqslant-\frac{1}{4} v F_{2}+c v^{-3} F_{1}^{3}+R e F_{1},
$$

as shown in Doering \& Gibbon (1995), to obtain

$$
\int_{t_{0}}^{t} \kappa_{2,1}\left(t^{\prime}\right) \mathrm{d} t^{\prime} \leqslant c v^{-2} \int_{t_{0}}^{t} F_{1} \mathrm{~d} t^{\prime}
$$

where small correction terms have been dropped. Hence the last two terms of (A 3) are determined by (A 4) alone. Using the lower bound (3.6) in the bad interval, inequality (A 3) becomes

$$
A_{n} \nu^{-1}\left[\kappa_{n, 1}^{-1}(t)-\kappa_{n, 1}^{-1}\left(t_{0}\right)\right] \geqslant c_{n, \mu} L^{-1} \int_{t_{0}}^{t} R e^{\lambda_{n} / \mu} \mathrm{d} t-c v^{-2} \int_{t_{0}}^{t} F_{1}\left(t^{\prime}\right) \mathrm{d} t^{\prime},
$$

where the $R e^{1-\lambda_{n} / \mu}$ term has been dropped as small. Now define the coefficients

$$
B_{n}=c_{n, \mu} R e^{\lambda_{n} / \mu}-c_{3} R e^{4}-c_{4} R e^{2},
$$

where $\lambda_{n}$ has already been defined in (2.5). Also define

$$
C_{n}=c_{1} R e^{4}-A_{n} L^{-1} \kappa_{n, 1}^{-1}\left(t_{0}\right)>0 .
$$

Using the estimate (A 4) in (A 7) gives

$$
A_{n} \kappa_{n, 1}^{-1}(t) \geqslant-L C_{n}+L^{-1} v B_{n} \Delta t .
$$

At high $R e$, to be sure that $B_{n}>0$ we need

$$
4 \mu<\lambda_{n} \text {. }
$$


In addition, singularities can only potentially occur when the right-hand side of (A 10) is negative; that is, in the interval $0 \leqslant \Delta t \leqslant(\Delta t)_{c}$ where

$$
(\Delta t)_{c}=c_{5} \frac{L^{2}}{v} R e^{4-\lambda_{n} / \mu} .
$$

Only dominant terms have been kept in (A 12). Now $\kappa_{n, 1} \geqslant \kappa_{n}$, which has a lower bound in (3.6), so to avoid violating this the bad interval itself cannot exist for longer than

$$
(\Delta t)_{b}=\frac{L^{2}}{v}\left(c_{5} R e^{4-\lambda_{n} / \mu}+c_{6} R e^{-2 \lambda_{n} / \mu}\right) .
$$

\section{REFERENCES}

BAtchelor, G. K. \& Townsend, A. A. 1949 The nature of turbulent flow at large wavenumbers. Proc. R. Soc. Lond. A 199, 238-255.

Caffarelli, L., Kohn, R. \& Nirenberg, L. 1982 Partial regularity of suitable weak solutions of the Navier-Stokes equations. Commun. Pure Appl. Maths 35, 771-831.

ChoE, H. J. \& Lewis, J. L. 2000 On the singular set in the Navier-Stokes equations. J. Funct. Anal. 175, 348-369.

Constantin, P. \& Folas, C. 1988 The Navier-Stokes Equations. Chicago University Press.

Doering, C. R. \& FoAis, C. 2002 Energy dissipation in body-forced turbulence. J. Fluid Mech. 467, 289-306.

Doering, C. R. \& Gibbon, J. D. 1995 Applied Analysis of the Navier-Stokes Equations. Cambridge University Press.

Doering, C. R. \& Gibbon, J. D. 2001 Scale separation \& regularity in the Navier-Stokes equations. In Intermittency in Turbulent Flows (ed. J. C. Vassilicos), pp. 470-479. Cambridge University Press.

Doering, C. R. \& Gibion, J. D. 2002 Bounds on moments of the energy spectrum for weak solutions of the three-dimensional Navier-Stokes equations. Physica D 165, 163-175.

Foias, C., Manley, O., Rosa, R. \& Temam, R. 2001 Navier-Stokes Equations \& Turbulence. Cambridge University Press.

FRISCH, U. 1995 Turbulence. Cambridge University Press.

Kuo, A. Y.-S. \& CoRrsin, S. 1971 Experiments on internal intermittency and fine-structure distribution functions in fully turbulent fluid. J. Fluid Mech. 50, 285-320.

Leray, J. 1934 Essai sur le mouvement d'un liquide visquex emplissant l'espace. Acta Math. 63, $193-248$.

Lin, F. 1998 A new proof of the Caffarelli, Kohn \& Nirenberg theorem. Commun. Pure Appl. Maths 51, 241-257.

MANDELBROT, B. 1976 Intermittent turbulence $\&$ fractal dimension: kurtosis $\&$ the spectral exponent $\frac{5}{3}+$ B. In Turbulence and Navier-Stokes Equations (ed. R. Temam). Lecture Notes in Maths, vol. 565, pp. 121-145. Springer.

Mandelbrot, B. 1982 The Fractal Geometry of Nature. Freeman, San Francisco.

Meneveau, C. \& Sreenivasan, K. 1991 The multifractal nature of turbulent energy dissipation. J. Fluid Mech. 224, 429-484.

SCHEFfer, V. 1976 Partial regularity of solutions to the Navier-Stokes equations. Pacific J. Maths 66, 535-552.

Sreenivasan, K. 1985 On the fine-scale intermittency of turbulence. J. Fluid Mech. 151, 81-103.

Sreenivasan, K. \& Meneveau, C. 1988 Singularities of the equations of fluid motion. Phys. Rev. A 38, 6287-6295.

Sulem, P.-L. \& Frisch, U. 1975 Bounds on energy flux for finite energy turbulence. J. Fluid Mech. 72, 417-424.

Temam, R. 1983 Navier-Stokes Equations \& Non-linear Functional Analysis. CBMS-NSF Regional Conference Series in Applied Mathematics, SIAM Press, Philadelphia. 